\title{
Some Results on Fixed and Best Proximity Points of Multivalued Cyclic Self-Mappings with a Partial Order
}

\author{
M. De la Sen \\ Institute of Research and Development of Processes, University of Basque Country, Campus of Leioa (Bizkaia, Apatado) 644, \\ 48080 Bilbao, Spain
}

Correspondence should be addressed to M. De la Sen; manuel.delasen@ehu.es

Received 17 October 2012; Revised 7 March 2013; Accepted 22 March 2013

Academic Editor: Abdul Latif

Copyright (C) 2013 M. De la Sen. This is an open access article distributed under the Creative Commons Attribution License, which permits unrestricted use, distribution, and reproduction in any medium, provided the original work is properly cited.

This paper is devoted to investigate the fixed points and best proximity points of multivalued cyclic self-mappings on a set of subsets of complete metric spaces endowed with a partial order under a generalized contractive condition involving a Hausdorff distance. The existence and uniqueness of fixed points of both the cyclic self-mapping and its associate composite self-mappings on each of the subsets are investigated, if the subsets in the cyclic disposal are nonempty, bounded and of nonempty convex intersection. The obtained results are extended to the existence of unique best proximity points in uniformly convex Banach spaces.

\section{Introduction}

Important attention is being devoted recently to the investigation of fixed points of self-mappings as well as to the investigation of associate relevant properties like, for instance, stability of the iterations [1-3] and existence and uniqueness of fixed points. On the other hand, the extension of those topics to the existence of either fixed points of multivalued self-mappings [1, 4-19], or common fixed points of several multivalued mappings or operators has received important attention; see, for example, [15-19] and references therein. This paper investigates some properties of fixed point and best proximity point results for multivalued cyclic selfmappings under a general contractive-type condition based on the Hausdorff metric between subsets of a metric space $[4,7-9]$ and which includes a particular case the contractive condition for contractive single-valued self-mappings, [1, 4$10]$ including the problems related to cyclic self-mappings, see for example, $[7,8,11]$ and references therein. This includes strict contractive cyclic self-mappings and Meir-Keeler type cyclic contractions, $[20,21]$. There is a rich background literature available on cyclic self-mappings and related fixed point and best proximity point results; see, for example, [22-30] and references therein. Some existing fixed point results on contractive single and multivalued self-mappings provided in $[1,4,5,9,10,31,32]$ and references therein, under various types of contractive conditions, have been revisited and extended in [4]. There is also a wide sample of fixed point type results available on fixed points and asymptotic properties of the iterations for self-mappings satisfying a number of contractive-type conditions while being endowed with partial order conditions. See, for instance, $[18,19]$, and references therein. The main objective of this paper is the investigation of fixed point/best proximity point results for multivalued cyclic self-mappings in complete metric spaces, or uniformly convex Banach spaces. Such multivalued cyclic self-mappings satisfy a contractive-type condition, which is specified on the Hausdorff metric, for all pairs of elements in the union of the subsets defining the cyclic disposal which are subject to a partial order.

\section{Properties of Distances and Fixed Points for Multivalued Cyclic Self-Mappings with a Partial Order}

Assume that $(X, d)$ is a metric space for a set $X$ endowed with some metric: $X \times X \rightarrow \mathbf{R}_{0+}$ with $\mathbf{R}_{0+}=\mathbf{R}_{+} \cup\{0\}$. Let $C L(X)$ be the family of all nonempty and closed subsets of the set $X$. If $A, B \in C L(X)$ then we can define $(C L(X), H)$ being the generalized hyperspace of $(X, d)$ equipped with the Hausdorff 
metric $H: C L(X) \rightarrow \mathbf{R}_{0+}$ induced by the metric $d: X \times X \rightarrow$ $\mathbf{R}_{0+}$ :

$$
H(A, B)=\max \left\{\sup _{x \in A} d(x, B), \sup _{y \in B} d(y, A)\right\}
$$

for two sets $A \subseteq X$ and $B \subseteq X$ which are finite if both sets are bounded and zero if they have the same closure. The distance between $A \subseteq X$ and $B \subseteq X$ is

$$
\begin{aligned}
D & =d(A, B)=\inf _{x \in A, y \in B} d(x, y) \\
& =\inf _{x \in A} d(x, B)=\inf _{y \in B} d(y, A) .
\end{aligned}
$$

Denote by $P(X), B(X)$, and $C B(X)$ the sets of nonempty, and nonempty, bounded and nonempty, and bounded and closed sets of $X$, respectively. The following relations hold:

$$
\begin{aligned}
D & \leq H(A, B) \\
& \leq \delta(A, B)=\delta(B, A)=\sup _{x \in A, y \in B} d(a, b) \\
& \leq \delta(A, B)+\delta(B, C) ; \quad \forall A, B, C \in B(X), \\
& {[(A, B \in C B(X)) \wedge H(A, B)<\varepsilon] } \\
& \Longrightarrow[\exists b \in B: d(a, b)<\varepsilon, \forall a \in A]
\end{aligned}
$$

and $\delta(A, B)=0$ if and only if $A=B=\{x\}$. Consider also a self-mapping $T: \bigcup_{i \in \bar{p}} A_{i} \rightarrow \bigcup_{i \in \bar{p}} A_{i}$, where $A_{i}$ are nonempty closed sets of $X ; \forall i \in \bar{p}=\{1,2, \ldots, p\}$, subject to the constraints $T\left(A_{i}\right) \subseteq A_{i+1}$ such that $A_{i p+j} \equiv A_{j}$ for any integer numbers $j \in[1, p-1) \cap \mathbf{Z}$ and $i \in \mathbf{Z}_{0+}=\mathbf{Z}_{+} \cup\{0\}$ with $\mathbf{R}_{0+}=\mathbf{R}_{+} \cup\{0\}$. If $p \geq 2$ then $T: \bigcup_{i \in \bar{p}} A_{i} \rightarrow \bigcup_{i \in \bar{p}} A_{i}$ is a $p$-cyclic self-mapping. If $p=1$ then $T: A_{1} \rightarrow A_{1}$ is, in particular, a self-mapping on $A_{1}$. We will also consider a partial order $\preceq$ on $X$ so that $(X, \preceq)$ is a partially ordered space and will assume, in general, that $T: \bigcup_{i \in \bar{p}} A_{i} \rightarrow \bigcup_{i \in \bar{p}} A_{i}$ is a multivalued $p$-cyclic self-mapping so that $A_{i} \ni x \rightarrow$ $T x(\neq \varnothing) \subset A_{i+1} ; \forall i \in \bar{p}, \forall x \in \bigcup_{i \in \bar{p}} A_{i}$. The subsequent result does not assume a contractive condition for each iteration on adjacent subsets of the contractive mapping but a global contractive condition for the cyclic mapping for iterations on multiple strips of the $p$ subsets $A_{i} \subset X ; i \in \bar{p}$. Therefore, the result that the distances between any two subsets being adjacent or not of [33] for nonexpansive self-mappings is not required.

If $T: \bigcup_{i \in \bar{p}} A_{i} \rightarrow \bigcup_{i \in \bar{p}} A_{i}$ is a multivalued $p$-cyclic selfmapping then the set $B P\left(A_{i}\right) \subset A_{i}$ will be said to be the set of best proximity points between $A_{i}$ to $A_{i+1}$ if $d\left(A_{i}, A_{i+1}\right)=$ $D_{i}=d(z, y)$ for all $z \in A_{i}$ and some $y \in T z$. This concept generalizes that of best proximity points of subsets of single valued cyclic self-mappings which is established as follows. If $T: A_{1} \cup A_{2} \rightarrow A_{1} \cup A_{2}$ is cyclic and single-valued then $x \in A_{1}$ and $T x \in A_{2}$ are best proximity point if $d\left(A_{1}, A_{2}\right)=$ $d(x, T x),[33,34]$. The following result extends a previous one for the case of noncyclic multivalued self-mappings, $[18,19]$.
Theorem 1. Let $(X, \leq)$ be a partially ordered space and $d: X \times$ $X \rightarrow \mathbf{R}_{0+}$ with $(X, d)$ being a complete metric space. Let $A_{i}$ be a set of $p(\geq 2)$ nonempty, bounded, and closed subsets of $X ; \forall i \in \bar{p}$ (i.e., $\left.A_{i} \in C B(X) ; \forall i \in \bar{p}\right)$ with $D_{i}=d\left(A_{i}, A_{i+1}\right)$; $\forall i \in \bar{p}$ and let $T: \bigcup_{i \in \bar{p}} A_{i} \rightarrow \bigcup_{i \in \bar{p}} A_{i}$ be a multivalued p-cyclic self-mapping on $\bigcup_{i \in \bar{p}} A_{i}$ satisfying.

(1) There exist $p$ real constants $k_{i} \in \mathbf{R}_{0+}$ satisfying $k=$ $\prod_{i \in \bar{p}}\left[k_{i}\right] \in[0,1)$ such that the following condition holds:

$$
H(T x, T y) \leq k_{i} d(x, y)+\left(1-k_{i}\right) D_{i}
$$

for any given $x \in A_{i}$ and $y \in A_{i+1}$ which fulfil $x \leq y$, $\forall i \in \bar{p}$.

(2) If $d(x, y)<d_{0}$ for some given $d_{0} \in \mathbf{R}_{+}, y \in T x$, and any given $x \in \bigcup_{i \in \bar{p}} A_{i}$, then $x \leq y$ with $y \in A_{j+1}$ if $x \in A_{j}$ for any given $j \in \bar{p}$.

(3) There are some $i \in \bar{p}$, some $x=x_{i} \in A_{i}$, and some $x_{i+1} \in T x_{i} \subset A_{i+1}$ such that $d\left(x_{i}, x_{i+1}\right)<d_{0 i}$ for some $d_{0 i}>D_{i}$.

(4)

$$
d_{0} \geq \max \left(\max _{j \in \bar{p}} d_{0 j}, \max _{j \in \bar{p}}\left(k_{j}\left(d_{0 j}-D_{j}\right)+D_{j}\right)\right) .
$$

Note that (6) implies that $d_{0 j} \leq \min \left(d_{0},\left(d_{0}-\left(1-k_{j}\right) D_{j}\right) / k_{j}\right)$; $\forall j \in \bar{p}$. Then, the following properties hold.

(i) There is a partially ordered subsequence $\widehat{S}_{i}=$ $\left\{x_{i+j+n_{k} p}\right\}_{n_{k} \in \mathbf{Z}_{0+}}$ of the partially ordered sequence $S\left(x_{i}\right)=\left\{x_{i+j}\right\}_{j \in \mathbf{Z}_{0+}}$, both of them of the first element $x_{i}$, with respect to the partial order $(X, \preceq)$, such that $x_{i+j+n_{k} p} \in Q_{i+j}$ for $j \in \bar{p} ; \forall k \geq k_{0}, n_{k} \in \mathbf{Z}_{0+}$ for some $k_{0} \in \mathbf{Z}_{0+}$ and the given $i \in \bar{p}$, where $Q_{i+j} \subseteq T x_{i+j-1} \subseteq$ $T^{j-1} x_{i} \subseteq A_{i+j}$, for any $j \in \overline{p-1} \cup\{0\}$ and the given $i \in \bar{p}$, are $p$ closed "quasi-proximity" sets in-between each pair of adjacent subsets of the multivalued p-cyclic self-mapping $T: \bigcup_{i \in \bar{p}} A_{i} \rightarrow \bigcup_{i \in \bar{p}} A_{i}$ such that

$$
\begin{aligned}
D_{i+j} & \leq d\left(x_{n p+i+j+1}, x_{n p+i+j}\right) \\
& \leq k_{i+j} D+\left(1-k_{i+j}\right) D=D, \\
& \forall j \in \overline{p-1} \cup\{0\}, \forall n \in \mathbf{Z}_{0+},
\end{aligned}
$$

where $D=\min _{j \in \bar{p}} D_{j}$ with $x_{n p+i+j} \in T x_{n p+i+j-1} \subseteq$ $A_{i+j} ; \forall j \in \overline{p-1} \cup\{0\}, \forall n \in \mathbf{Z}_{0+}$ for the given $i \in \bar{p}$.

(ii) If $D_{j}=D ; \forall j \in \bar{p}$ then any partially ordered sequence $S\left(x_{i}\right)$ of first element $x=x_{i} \in A_{i}$ fulfills

$$
\exists \lim _{n \rightarrow \infty} d\left(x_{n p+i+j+1}, x_{n p+i+j}\right)=D
$$

$\forall j \in \bar{p}$ and the given $i \in \bar{p}$, and $x_{n p+j+1} \in T x_{n p+j} \subseteq$ $A_{i+j+1} ; \forall j \in \bar{p}$ (i.e., $x_{n p+j+1} \in A_{i+j+1}$ if $0 \leq j \leq p-i-1$ and $x_{n p+j+1} \in A_{j-p+i+1}$ if $\left.p-i<j \leq p-1\right), \forall n \in \mathbf{Z}_{0+}$. 
Let $B P\left(A_{j}\right)$ be the set of best proximity points between $A_{j}$ and $A_{j+1} ; \forall j \in \bar{p}$. Then, there is a sequence $\left\{z_{n}^{(j)}\right\} \subset$ $B P\left(A_{j}\right) ; \forall j \in \bar{p}$ such that the following limit exists:

$$
\begin{aligned}
\lim _{n \rightarrow \infty} d\left(x_{n p+j+1}, z_{n}^{(j)}\right)=D ; & \forall j \in \bar{p} \\
\text { with } x_{n p+j+1} \in T x_{n p+j} ; & \forall n \in \mathbf{Z}_{0+}
\end{aligned}
$$

(iii) If assumption (3) is removed and (6) in assumption (4) is replaced by the stronger condition

(5)

$$
\begin{aligned}
& d_{0}>\max \left(\max _{j \in \bar{p}}\left(D_{j}+\operatorname{diam}\left(A_{j}\right)\right),\right. \\
&\left.\max _{j \in \bar{p}}\left(k_{j}\left(d_{0 j}-D_{j}\right)+D_{j}\right)\right)
\end{aligned}
$$

then, properties (i)-(ii) hold for any $x \in \bigcup_{i \in \bar{p}} A_{i}$.

Proof. Let $x_{i} \in A_{i}$ for the given $i \in \bar{p}$ which satisfy assumption (3). Then, from such an assumption, there is $x_{i+1} \in T x_{i}$, which is also in $A_{i+1}$, since $T x \in A_{i+1}$ for any $x \in \bigcup_{i \in \bar{p}} A_{i}$, such that $d\left(x_{i}, x_{i+1}\right)<d_{0 i} \leq d_{0}$. Thus, $x_{i} \preceq x_{i+1}$ from assumption (2), since $d_{0 i} \leq d_{0}$. From (6) and assumptions (1)-(2) by considering the distance between adjacent subsets,

$$
\begin{aligned}
D_{i} & \leq H\left(T x_{i}, T\right) \\
& \leq k_{i} d\left(x_{i}, x_{i+1}\right)+\left(1-k_{i}\right) D_{i} \\
& <k_{i} d_{0 i}+\left(1-k_{i}\right) D_{i} \leq d_{0 i} \leq d_{0},
\end{aligned}
$$

since $D_{i}<d_{0 i} \leq\left(1 / k_{i}\right)\left[\left(k_{i}-1\right) D_{i}+d_{0}\right]$ from assumptions (3)-(4). From assumption (2) and (11), there is $x_{i+2} \in T x_{i+1} \subset$ $A_{i+2}$ such that $x_{i+1} \preceq x_{i+2}$, and then $d\left(x_{i+1}, x_{i+2}\right)<d_{0, i+1}$, and $d\left(x_{i+1}, x_{i+2}\right) \leq H\left(T x_{i}, T x_{i+1}\right)<d_{0 i} \leq d_{0}$. Then, one gets from (11) and assumption (4):

$$
\begin{aligned}
D_{i+1} \leq & H\left(T x_{i+1}, T x_{i+2}\right) \\
\leq & k_{i+1} d\left(x_{i+1}, x_{i+2}\right)+\left(1-k_{i+1}\right) D_{i+1} \\
\leq & k_{i+1} d_{0, i+1}+\left(1-k_{i+1}\right) D_{i+1} \\
< & k_{i+1} d_{0, i+1} \\
& +\left(1-k_{i+1}\right)\left[d_{0}-k_{i+1}\left(d_{0, i+1}-D_{i+1}\right)\right]
\end{aligned}
$$

$$
\begin{aligned}
\leq & d_{0}+k_{i+1} d_{0}-k_{i+1} d_{0} \\
& +\left(1-k_{i+1}\right) k_{i+1}\left(D_{i+1}-d_{0 i+1}\right)<d_{0} \\
d\left(x_{i+2}, x_{i+3}\right) \leq & H\left(T x_{i+1}, T x_{i+2}\right) \\
\leq & k_{i+1} d\left(x_{i+1}, x_{i+2}\right) \\
& +\left(1-k_{i+1}\right) D_{i+1} \\
\leq & k_{i+1}\left[k_{i} d\left(x_{i}, x_{i+1}\right)+\left(1-k_{i}\right) D_{i}\right] \\
& +\left(1-k_{i+1}\right) D_{i+1} \\
\leq & k_{i+1} k_{i} d\left(x_{i}, x_{i+1}\right) \\
& +\left[k_{i+1}\left(1-k_{i}\right)+\left(1-k_{i+1}\right)\right] \max _{i \in \bar{p}} D_{i}<d_{0} .
\end{aligned}
$$

Again, from assumption (2), there is $x_{i+3} \in T x_{i+2}$ such that $x_{i} \preceq x_{i+1} \preceq x_{i+2} \preceq x_{i+3}$. Now, proceeding by complete induction with (12a) from $j=0$ to $j=p-1$, it follows that the existence of a partially ordered space $x_{i} \preceq x_{i+1} \preceq$ $x_{i+2} \preceq x_{i+p-2}$ implies, from assumption (2), the existence of the partially ordered space $x_{i} \preceq x_{i+1} \preceq x_{i+2} \preceq \cdots \preceq x_{i+p-1}$ satisfying $x_{i+j} \in T x_{i+j-1} \subseteq A_{i+j} ; \forall j \in \bar{p}$ with $A_{k p+j} \equiv$ $A_{j} ; \forall k \in \bar{p}$. Also, proceeding recursively with (12b), one concludes, if $k=\prod_{i \in \bar{p}}\left[k_{i}\right] \in[0,1)$ and $D=\max _{i \in \bar{p}} D_{i}$, that there is a partially ordered sequence $x_{i} \preceq x_{i+1} \preceq x_{i+2} \preceq \cdots \preceq$ $x_{i+p-1} \preceq x_{i+p}$ such that $x_{i+j} \in T x_{i+j-1} \subseteq T\left(A_{i+j-1}\right) \subseteq A_{i+j}$; $\forall j \in \bar{p}$, and

$$
d\left(x_{i+p+1}, x_{i+p}\right) \leq k d\left(x_{i}, x_{i+1}\right)+(1-k) D
$$

so that there is a partially ordered sequence $x_{i} \preceq x_{i+1} \preceq x_{i+2} \preceq$ $\cdots \preceq x_{i+p-1} \preceq x_{i+n p} \preceq \cdots$ such that $x_{i+j} \in T x_{i+n p+j-1} \subseteq$ $T\left(A_{i+j}\right) ; \forall j \in \overline{p-i}$ :

$$
\begin{aligned}
D_{i+1} & \leq d\left(x_{n p+i+2}, x_{n p+i+1}\right) \\
& \leq H\left(T x_{i+n p+1}, T x_{n p+i}\right)+\left(1-k_{i}\right) D_{i} \\
& \leq \lim \sup \left[k ^ { n } d \left(\begin{array}{c}
\left.\left.x_{i}, x_{i+1}\right)+\left(1-k^{n}\right) D\right]=D ; \\
n \rightarrow \infty
\end{array}\right.\right.
\end{aligned}
$$

$\forall n \in \mathbf{Z}_{+}$

so that

$$
\begin{aligned}
D_{i+j} \leq & d\left(x_{n p+i+j+1}, x_{n p+i+j}\right) \\
\leq & k_{i+j} D+\left(1-k_{i+j}\right) D=D ; \\
& \forall j \in \overline{p-1} \cup\{0\}, \forall n \in \mathbf{Z}_{0+} .
\end{aligned}
$$

Then, from (15), there are $p$ closed "quasi-proximity" sets $Q_{i} \subseteq A_{i}, Q_{i+1} \subseteq T x_{i} \subseteq A_{i+1}, Q_{i+p-1} \subseteq T^{p-1} x_{i} \subseteq$ $A_{i+p-1}$ between each pair of adjacent subsets of the cyclic self-mapping $T: \bigcup_{i \in \bar{p}} A_{i} \rightarrow \bigcup_{i \in \bar{p}} A_{i}$ in view of (14), such 
that there is a partially ordered subsequence $\left\{x_{i+j+n_{k} p}\right\}_{n_{k} \in \mathbf{Z}_{+}}$ of the partially ordered sequence $x_{i} \preceq x_{i+1} \preceq x_{i+2} \preceq \cdots \preceq$ $x_{i+p-1} \preceq x_{i+n p} \preceq \cdots$, being subject to $x_{i+j+n_{k} p} \in Q_{i+j}$ for $\forall j \in \overline{p-1} \cup\{0\} ; \forall k \geq k_{0}$, some $k_{0} \in \mathbf{Z}_{0+}$. Thus, (7) holds and then the property (i) has been proven. The relation (8) of property (ii) for $j=0$ is a direct consequence of property (i). From (8), it is also proven that the sequence $S_{i}$ of first element $x_{i} \in A_{i}$ in property (i) satisfies the following property $\exists \lim _{n \rightarrow \infty} d\left(x_{j+n p+1}, x_{j+n p}\right)=D ; \forall j \in \bar{p}$. Assume not, then, it follows that

$$
\begin{aligned}
D \leq & \liminf _{n \rightarrow \infty} d\left(x_{i+j+n p+1}, x_{i+j+n p}\right) \\
\leq & \limsup _{n \rightarrow \infty} d\left(x_{i+j+n p+1}, x_{i+j+n p}\right) \\
\leq & k_{j} \lim _{n \rightarrow \infty} d\left(x_{i+j+n p}, x_{i+j+n p-1}\right) \\
& +\left(1-k_{j}\right) D \leq k_{j} D+\left(1-k_{j}\right) D=D ;
\end{aligned}
$$

$\forall j \in \overline{p-1} \cup\{0\}$ and the given $i \in \bar{p}$ so that, by using complete induction, $\exists \lim _{n \rightarrow \infty} d\left(x_{i+j+n p+1}, x_{i+j+n p}\right)=D$; $\forall j \in \overline{p-1} \cup\{0\}$ and the given $i \in \bar{p}$ with $\left\{x_{k}\right\}_{k \geq i}$ being partially ordered with respect to $(\mathrm{X}, \preceq)$, that is, $x_{i+k}\left(\in T x_{i+k-1} \subseteq\right.$ $\left.A_{i+k}\right) \preceq x_{i+k+1}\left(\in T x_{i+k} \subseteq A_{i+k+1}\right) ; \forall k \in \mathbf{Z}_{0+}$ and we can then reformulate the above limits of the distances as $\exists \lim _{n \rightarrow \infty} d\left(x_{i+j+n p+1}, x_{i+j+n p}\right)=D ; \forall j \in \overline{p-1} \cup\{0\}$ for the given $i \in \bar{p}$.

The remaining proof of property (ii) follows by contradiction. Suppose that the limit (9) does not exist for some sequence $\left\{z_{n}^{(j)}\right\} \subset B P\left(A_{j}\right)$ for some $j \in \bar{p}$. Since $\left\{z_{n}^{(j)}\right\} \subset$ $B P\left(A_{j}\right), \lim \inf _{n \rightarrow \infty} d\left(x_{n p+j+1}, z_{n}^{(j)}\right)<D$ is impossible in the case that $\lim \inf _{n \rightarrow \infty} d\left(x_{n p+i+1}, z_{n}^{(j)}\right)$ would not exist for some $j \in \bar{p}$. Then,

$$
\begin{aligned}
& \liminf _{n \rightarrow \infty} d\left(x_{n p+j+1}, z_{n}^{(j)}\right)=D \\
& \quad \neq \liminf _{k \rightarrow \infty} d\left(x_{n_{k} p+j+1}, z_{n_{k}}^{(j)}\right)>D \\
& \quad=d\left(A_{j}, A_{j+1}\right)=d(z, y)
\end{aligned}
$$

for some $z \in B P\left(A_{j}\right), y \in T z \subset B P\left(A_{j+1}\right)$, since $A_{j}$ and $A_{j+1}$ are boundedly compact for all $j \in \bar{p}$ since they are bounded and closed and, then, compact, $[7,8]$. This leads to a contradiction, since $\left\{z_{n}^{(j)}\right\} \subset B P\left(A_{j}\right)$ and $x_{n p+j+1} \in T_{n p+j} \subset$ $A_{j+1} ; \forall j \in \bar{p}$. The property (ii) has been proven.

If assumption (3) is removed, while $d_{0}$ satisfies the stronger constraint (10), then there are infinitely many sequences $S(x)$ for any arbitrary first element $x \in \bigcup_{i \in \bar{p}} A_{i}$, in the partial order $\preceq$, of an iterated sequence through $T$ for which property (i) and thus property (ii) both hold since $d(x, y)=d(x, T x)<d_{0}$ from assumption (2). Hence, property (iii) follows so that the theorem has been fully proven.

Note that (5) is not guaranteed to be a cyclic contractive condition for each restricted map $T:\left(\bigcup_{j \in \bar{p}} A_{j}\right) \mid A_{i} \rightarrow$ $\left(\bigcup_{j \in \bar{p}} A_{j}\right) \mid A_{i+1}$, since all the constants are not required to be less than one in (5), and furthermore, (5) and assumption (3) are fulfilled for some first element $x_{i} \in A_{i}, x_{i+1} \in T x_{i} \subseteq$ $A_{i+1}$ and some given $i \in \bar{p}$ in the partial order $(X, d)$. Note also that sequences fulfilling the partial order of Theorem 1 can always be built through iterations with the multivalued $p$-self-mapping for any arbitrarily chosen $A_{i}$ for any $i \in \bar{p}$ from (6) characterizing assumption (4) of Theorem 1 . The subsequent particular case of Theorem 1 applies when all the iterations between the cyclic disposal satisfy a cyclic contractive condition, that is, $k_{i}<1 ; \forall i \in \bar{p}$.

Note that Theorem 1(iii) also holds in the particular case that the partial order is a total order for all pairs in any Cartesian product $A_{i} \times A_{i+1}$ of adjacent subsets $A_{i} \subset X ; \forall i \in$ $\bar{p}$, since both elements of any ordered pair $(x, y) \in A_{i} \times A_{i+1}$, $y \in T x ; \forall i \in \bar{p}$ are comparable with respect to the partial order $\preceq$. Theorem 1(iii) establishes that any element in any subset $A_{i} \subset X ; \forall i \in \bar{p}$ is a first element of a nondecreasing (i.e., partially ordered) sequence with respect to the partial order $\preceq$ which fulfils properties (i)-(ii) of Theorem 1 .

Theorem 2. In addition to assumptions (1)-(4) of Theorem 1, assume, furthermore,

(6) $D_{j}=D=0 ; \forall j \in \bar{p}$ (i.e., $\left.\bigcap_{j \in \bar{p}} A_{j} \neq \varnothing\right)$;

(7) the limit $x$ of any converging nondecreasing sequence $\left\{x_{n}\right\}_{n \in \mathbf{Z}_{0+}}$ is comparable to each $x_{n} ; \forall n \in \mathbf{Z}_{0+}$ in the partial order $(X, \preceq)$, that is,

$$
\begin{aligned}
& {\left[x_{n} \preceq x\left(\neq x_{n}\right) \text { for } x \in A_{j}, x_{n} \in A_{j}, \forall j \in \bar{p}, \forall n \in \mathbf{Z}_{0+}\right]} \\
& \Longrightarrow H\left(T x, T x_{n}\right)>k_{i} d\left(x, x_{n}\right) .
\end{aligned}
$$

Then, there is a sequence $\left\{x_{n p+i+j}\right\}_{n \in \mathbf{Z}_{0+}}$ satisfying $x_{n p+i+j} \in$ $T^{n p+j} x_{i}$ for some given initial element $x=x_{i} \in A_{i}$ and some given $i \in \bar{p} ; \forall j \in \overline{p-1} \cup\{0\}$ which is non-decreasing and ordered with respect to the partial order $(X, \preceq)$ and fulfils the following properties.

(i) $\exists \lim _{n \rightarrow \infty} d\left(x_{n p+i+j+2}, x_{n p+i+j+1}\right)=0 ; j \in \overline{p-1} \cup\{0\}$ and the given $i \in \bar{p}$ with $x_{n p+j+2} \in T x_{n p+j+1} ; j \in$ $\overline{p-1} \cup\{0\}, \forall n \in \mathbf{Z}_{0+}$ and the sequence $\left\{x_{n p+i+j}\right\}_{n \in \mathbf{Z}_{0+}}$ is a Cauchy sequence; $j \in \overline{p-1} \cup\{0\}$.

(ii) The sequence $\left\{x_{n p+i+j}\right\}_{n \in \mathbf{Z}_{0+}}$ for any $j \in \overline{p-1} \cup\{0\}$ and the given $i \in \bar{p}$ converge to a limit $\bar{x}$ in $\bigcap_{j \in \bar{p}} A_{j}$, which is a fixed point of the composite self-mapping $\widehat{T}_{j}$ : $A_{j} \rightarrow A_{j}$, where $\widehat{T}_{j}=T^{p}=T \circ T \circ \cdots T$ ( $p$ times $)=$ $T^{p} \mid A_{j}$ of domain $A_{j} ; \forall j \in \bar{p}$ and also a fixed point of the self-mapping $T: \bigcup_{i \in \bar{p}} A_{i} \rightarrow \bigcup_{i \in \bar{p}} A_{i}$, that is, $\bar{x} \in \widehat{T}_{j} \bar{x}\left(\subseteq \bigcap_{j \in \bar{p}} A_{j}\right)$ and $\bar{x} \in T^{p} \bar{x}\left(\subseteq \bigcap_{j \in \bar{p}} A_{j}\right)$; $\forall j \in \bar{p}$.

(iii) If, in addition, $(X, d)$ is a convex metric space, what holds, in particular, if $X$ is a Euclidean vector space and $d: X \times X \rightarrow \mathbf{R}_{0+}$ is the Euclidean metric, and 
$\bigcap_{j \in \bar{p}} A_{j}$ is convex, then $\bar{x} \in T \bar{x}\left(\subseteq \bigcap_{j \in \bar{p}} A_{j}\right)$ is the unique fixed point of $T: \bigcup_{i \in \bar{p}} A_{i} \rightarrow \bigcup_{i \in \bar{p}} A_{i}$ and $\widehat{T}_{j}: A_{j} \rightarrow A_{j} ; \forall j \in \bar{p}$ and also the unique fixed point of $T^{p}: \bigcup_{i \in \bar{p}} A_{i} \rightarrow \bigcup_{i \in \bar{p}} A_{i}$.

(iv) If assumption (4) of Theorem 1 is replaced by assumption (5) then properties (i)-(iii) hold for any $x \in$ $\bigcup_{i \in \bar{p}} A_{i}$.

(v) If $X$ is a Euclidean vector space then property (iii) holds also if the condition of $(X, d)$ being a convex metric space is removed.

Proof. The property (i) follows from Theorem 1 when $D_{j}=$ $D=0 ; \forall j \in \bar{p}$. To address the proof of property (ii), it is first proven that $\left\{x_{n p+j}\right\}_{i \in \mathbf{Z}_{0+}}$ is a Cauchy sequence in $X ; \forall j \in \bar{p}$. Take $m \in \mathbf{Z}_{+}$so that one gets from (10) that

$$
\begin{aligned}
& d\left(x_{(n+m+1) p+i+j}, x_{(n+m) p+i+j}\right) \\
& \quad \leq k d\left(x_{(n+m) p+i+j}, x_{(n+m-1) p+i+j}\right) \\
& \leq \cdots \leq k^{m} d\left(x_{n p+i+j}, x_{(n+1) p+i+j}\right)
\end{aligned}
$$

for some $x=x_{i} \in A_{i}, \forall j \in \overline{p-1} \cup\{0\}$ for any given $i \in \bar{p}$ from assumption (1) of Theorem 1 , where $k=\prod_{i=1}^{p} k_{i}$. Then, $d\left(x_{(n+m+1) p+i+j}, x_{(n+m) p+i+j}\right) \rightarrow 0$ as $m \rightarrow \infty ; \forall j \in \overline{p-1} \cup$ $\{0\}$. Thus, $\left\{x_{n p+i+j}\right\}$ is a Cauchy sequence for the given $i \in \bar{p}$; $\forall j \in \overline{p-1} \cup\{0\}$. Then, $\left\{x_{n p+j}\right\}$ is a Cauchy sequence; $\forall j \in$ $[i, p+i]$. Then, such a sequence converges to some $\bar{x}_{i+j} \in$ $T \bar{x}_{i+j-1} \cap A_{i+j} ; \forall j \in \overline{p-1} \cup\{0\}$ and any given $i \in \bar{p}$, such that $x_{n p+i+j} \preceq \bar{x}_{i+j} ; \forall n \in \mathbf{Z}_{0+}$ from (18), since all the elements of the generated non-decreasing sequence in the partial order $(X, \preceq)$ are comparable from $(18)$ and $(X, d)$ is complete. The property (i) has been proven.

To prove property (ii), assume that there are two distinct limits $\bar{x}_{i} \in T \bar{x}_{i-1} \cap A_{i}$ and $\bar{x}_{j} \in T \bar{x}_{j-1} \cap A_{j}$ for some distinct $i, j$ in $\bar{p}$. Since the restricted composite self-mapping $\widehat{T}_{j}\left(\equiv T^{p} \mid\right.$ $\left.A_{j}\right): A_{j} \rightarrow A_{j}$ is a cyclic contraction on the nonempty closed set $A_{j}$ for any $j \in \bar{p}$, then we can built uniquely a restricted composite self-mapping $\widehat{T}_{j}: A_{i} \rightarrow A_{i}$ defining the partially ordered sequence $\left\{x_{n p+i}\right\}$, with first element $x=$ $x_{i} \in A_{\mathrm{i}}$, which converges to $\bar{x}_{i}$ as $n \rightarrow \infty$, since such a restricted composite self-mapping satisfies also assumptions (1)-(2) of Theorem 1 . Then, we can proceed, in the same way, with $\widehat{T}_{j}: A_{j} \rightarrow A_{j}$ generating $\left\{x_{n p+j}\right\}$ converging to $\bar{x}_{j} \neq \bar{x}_{i}$ as $n \rightarrow \infty$ for any $j \in \bar{p}$. Both such composite self-mappings are Lipschitz-continuous, since they are contractive with the Lipschitz constant being the contractive constant $k<1$, so that the limit of the distance can be permuted with the distance of the limits. Then, since $\bar{x}_{\ell}=\widehat{T}_{j} \bar{x}_{\ell}$ for $\ell=i, j$ and since $\bar{x}_{\ell}$ is a fixed point of $\widehat{T}_{\ell}: A_{\ell} \rightarrow A_{\ell}$ for $\ell=i, j(\neq i) \epsilon$ $\bar{p}$, the following contradiction holds to the existence of two distinct fixed points $\bar{x}_{i}\left(\in \widehat{T}_{i}^{n} \bar{x}_{i}\right) \neq \bar{x}_{j}\left(\epsilon \widehat{T}_{j}^{n} \bar{x}_{j}\right)$ for some $\ell=$ $i, j(\neq i) \in \bar{p} ; \forall n \in \mathbf{Z}_{0+}$ :

$$
\begin{aligned}
0 & =H\left(\lim _{n \rightarrow \infty} \widehat{T}_{i}^{n} \bar{x}_{i}, \lim _{n \rightarrow \infty} \widehat{T}_{j}^{n} \bar{x}_{j}\right) \\
& =H\left(\lim _{n \rightarrow \infty} T^{n p} \bar{x}_{i}, \lim _{n \rightarrow \infty} T^{n p} \bar{x}_{j}\right) \\
& =\lim _{n \rightarrow \infty} H\left(T^{n p} \bar{x}_{i}, T^{n p} \bar{x}_{j}\right) \\
& \leq \lim _{n \rightarrow \infty}\left(k^{n}\right) d\left(\bar{x}_{i}, \bar{x}_{j}\right)=0 \Longrightarrow \bar{x}_{i}=\bar{x}_{j} \quad \text { for } i, j(\neq i) \in \bar{p} .
\end{aligned}
$$

Since any existing fixed point in $A_{i} \cap A_{j}$ of $\widehat{T}_{\ell}\left(=T^{p} \mid A_{\ell}\right)$ : $A_{\ell} \rightarrow A_{\ell}$ for $\ell=i, j ; \forall i, j(\neq i) \in \bar{p}$ is comparable in the partial order $(X, \preceq)$ to any element of $A_{i} \cap A_{j}, \operatorname{diam}\left(A_{i} \cap\right.$ $\left.A_{j}\right)<d_{0}$ and $\bar{x}_{i} \in \widehat{T}_{i} \bar{x}_{i} \subseteq A_{i}, \bar{x}_{j} \in \widehat{T}_{j} \bar{x}_{j} \subseteq A_{j} ; \forall i, j(\neq i) \epsilon$ $\bar{p}$. Assuming, with no loss in generality, that $\bar{x}_{i} \preceq \bar{x}_{j}$, one can build, from the assumptions of Theorem 1 and the current comparability assumption (7), a nondecreasing, converging and partially ordered sequence:

$$
z_{1}=\bar{x}_{i} \preceq z_{2}=\bar{x}_{j} \preceq \cdots \preceq z_{n} \cdots \preceq z=\lim _{n \rightarrow \infty} z_{n}
$$

such that

$$
z_{2 n+1} \in T^{(2 n+1) p} \subseteq A_{i}, \quad z_{2 n} \in T^{(2 n+j-i) p} \subseteq A_{j}, \forall n \in \mathbf{Z}_{0+} .
$$

Thus, $\lim _{n \rightarrow \infty} T^{n p} \bar{x}_{i}\left(\subseteq A_{i}\right)=\lim _{n \rightarrow \infty} T^{n p} \bar{x}_{j}\left(\subseteq A_{j}\right)$ for any $i, j(\neq i) \in \bar{p}$. Since, $A_{i}$ and $A_{i}$ are closed and nonempty for any distinct $i, j$ in $\bar{p}$, then $z \in A_{i} \cap A_{j}$. Since the pair $(i, j)$ is arbitrary and the set $\bigcap_{i \in \bar{p}} A_{i}$ is nonempty and closed, then $z \in \widehat{T}_{j} z\left(\subseteq \bigcap_{i \in \bar{p}} A_{i}\right)$ for any $j \in \bar{p}$. Then, $z \in \widehat{T}_{i} z$ is a fixed point of $\widehat{T}_{i}: A_{i} \rightarrow A_{i} ; \forall i \in \bar{p}$. But, since $\bar{x}_{i} \in \widehat{T}_{i} \bar{x}_{i}$ is a fixed point of $\widehat{T}_{i}: A_{i} \rightarrow A_{i}$, it cannot converge through an iterated sequence to another distinct fixed point of the same self-mapping or to be distinct of it. Thus, $\bar{x}=\bar{x}_{i}=\bar{x}_{j}$ is a fixed point of the restricted composite self-mapping of $\widehat{T}_{i}: A_{i} \rightarrow A_{i} ; \forall i \in \bar{p}$ which is in the closed nonempty set $\bigcap_{j \in \bar{p}} A_{j}$. Then, $T^{p n} \bar{x}=T^{p} \bar{x}=\operatorname{Fix}\left(T^{p}\right) \subseteq \in \bigcap_{i \in \bar{p}} A_{i} ; \forall n \in \mathbf{Z}_{+}$. Also, note that, since $\bar{x} \preceq \bar{x}, \bar{x} \in T \bar{x}$ and $D_{j}=0, \forall j \in \bar{p}$, one gets from (10) that

$$
\begin{aligned}
0 & =d\left(\bar{x}, T^{p+1} \bar{x}\right)=H\left(T \bar{x}, T^{p+1} \bar{x}\right) \\
& \leq k_{j} d(\bar{x}, \bar{x})=k_{j} d\left(\bar{x}, T^{p} \bar{x}\right)=0,
\end{aligned}
$$

since $\bar{x} \in T^{p+1} \bar{x}$ from $H\left(T \bar{x}, T^{p+1} \bar{x}\right)=0$. Thus, $\operatorname{Fix}\left(T^{p}\right)=$ $\operatorname{Fix}\left(\widehat{T}_{i}^{p}\right) \subseteq \operatorname{Fix}\left(T^{p+1}\right) \subseteq T \bar{x}$.

It remains to be proven that $\bar{x} \in T \bar{x} \subseteq \bigcap_{j \in \bar{p}} A_{j}$ is the unique fixed point of $T: \bigcup_{i \in \bar{p}} A_{i} \rightarrow \bigcup_{i \in \bar{p}} A_{i}$, since $d_{0}>$ $\operatorname{diam}\left(A_{i} \cap A_{j}\right) \geq \operatorname{diam}\left(\bigcap_{k \in \bar{p}} A_{k}\right)$ and any existing fixed point in $\bigcap_{k \in \bar{p}} A_{k}$ is comparable, with respect to the partial order $(X, \preceq)$, to any element of $\bigcap_{k \in \bar{p}} A_{k}$. This is a consequence of 
assumption (2) of Theorem 1 , so that $d\left(\bar{x}_{i}, T^{n p+j} \bar{x}_{i+j}\right) \rightarrow 0$ as $n \rightarrow \infty$, and

$$
\begin{aligned}
d\left(\bar{x}_{i}, T^{n p+j} \bar{x}_{i+j}\right) & \leq H\left(T^{n p} \bar{x}_{i}, T^{n p+j} \bar{x}_{i+j}\right) \\
& \leq k^{n}\left(\prod_{\ell=i}^{i+j-1}\left[k_{\ell}\right]\right) d\left(\bar{x}_{i}, T^{j-1} \bar{x}_{i+j}\right) \\
& <d\left(\bar{x}_{i}, \bar{x}_{i+j}\right) ; \quad \forall n \in \mathbf{Z}_{0+},
\end{aligned}
$$

if $\bar{x}_{i}\left(\neq x_{i+j}\right) \notin T^{j-1} \bar{x}_{i+j}$, since $k<1$, and then $k^{n}\left(\prod_{\ell=i}^{i+j-1} k_{\ell}\right)<$ $1 ; \forall j \in \bar{p}, \forall n\left(\geq n_{0}\right)$ and some $n_{0} \in \mathbf{Z}_{+}$. Thus, $\bar{x}_{i} \in T \bar{x}_{i} \subseteq$ $T^{p n} \bar{x}_{i} \subseteq A_{i}$ and $\bar{x}_{i+j} \in T \bar{x}_{i+j} \subseteq T^{p n+j} \bar{x}_{i+j}\left(=T^{j} \bar{x}_{i+j}\right) \subseteq A_{i+j}$.

Thus, $\bar{x}_{i} \in T \bar{x}_{i} \Rightarrow \bar{x}_{i+j} \in T^{j} \bar{x}_{i+j}$. It can be also proven in the same way by interchanging the roles of both fixed points that $\bar{x}_{i+j} \in T^{j} \bar{x}_{i+j} \Rightarrow \bar{x}_{i} \in T \bar{x}_{i}$. As a result, $T^{j} \bar{x}_{i+j}=T \bar{x}_{i}=$ $\operatorname{Fix}(T) \subseteq \bigcap_{i \in \bar{p}} A_{i}$.

On the other hand, since any fixed point of the selfmapping $T: \bigcup_{i \in \bar{p}} A_{i} \rightarrow \bigcup_{i \in \bar{p}} A_{i} ; \forall i \in \bar{p}$ has to be also a fixed point of each restricted composite self-mapping $T_{j}$ : $A_{j} \rightarrow A_{j}, \forall j \in \bar{p}$, which is a unique $\bar{x} \in T \bar{x}, \forall i \in \bar{p}$, then, $\operatorname{Fix}(T)=\operatorname{Fix}\left(T^{p}\right)=\operatorname{Fix}\left(T_{j}^{p}\right) \subseteq \bigcap_{i \in \bar{p}} A_{i} ; \forall j \in \bar{p}$. Hence, property (ii) is proven.

The property (iii) is proven as follows. Assume that there are $\bar{x}_{i} \in T \bar{x}_{1}\left(\subseteq \bigcap_{j \in \bar{p}} A_{j}\right)$ for $i=1,2$ with $\bar{x}_{1} \neq \bar{x}_{2}$, since it has been proven that $T \bar{x}_{1}=T \bar{x}_{2}$ if $\bar{x}_{i} \in \operatorname{Fix}(T) ; i=1,2$. Then, since $(X, d)$ is a convex metric space (which is guaranteed, in particular, under a sufficiency-type condition, if the vector space $X$ is Euclidean and the metric is the Euclidean norm) and $\bigcap_{j \in \bar{p}} A_{j}$ is convex, there is a sequence $\left\{\bar{x}_{1}^{(n)}\right\} \subset\left[\bar{x}_{1}, \bar{x}_{2}\right] \subset$ $\bigcap_{j \in \bar{p}} A_{j}$, for some $\bar{x}_{1}^{(1)} \in T \bar{x}_{1}$ being such that $d\left(\bar{x}_{1}, \bar{x}_{1}^{(1)}\right)=$ $d\left(\bar{x}_{2}, \bar{x}_{1}^{(1)}\right)=d\left(\bar{x}_{1}, \bar{x}_{2}\right) / 2$, fulfilling

$$
\begin{aligned}
0 & =d\left(\bar{x}_{1}^{(n+1)}, \bar{x}_{2}\right) \\
& \leq d\left(\bar{x}_{1}^{(n)}, \bar{x}_{2}\right) \\
& \leq 2^{n} d\left(\bar{x}_{1}^{(n)}, \bar{x}_{2}\right) \\
& =2 d\left(\bar{x}_{1}^{(1)}, \bar{x}_{2}\right) \\
& \leq H\left(T \bar{x}_{1}, T \bar{x}_{2}\right) \\
& =H\left(T^{p} \bar{x}_{1}, T^{p} \bar{x}_{2}\right)=0 \\
& \leq k d\left(\bar{x}_{1}, \bar{x}_{2}\right)<d\left(\bar{x}_{1}, \bar{x}_{2}\right)<d_{0} ;
\end{aligned}
$$

$\forall n \in \mathbf{Z}_{+}$such $\bar{x}_{1}^{(1)} \in T \bar{x}_{1}$ always exists, since $\bar{x}_{1}=\bar{x}_{1}^{(0)} \preceq \bar{x}_{2}$, since all elements in $\bigcap_{j \in \bar{p}} A_{j}$ are pair wise comparable by hypothesis, $T \bar{x}_{1}=T \bar{x}_{2}, \bar{x}_{1,2} \in T \bar{x}_{1},\left[\bar{x}_{1}, \bar{x}_{2}\right] \subset \bigcap_{j \in \bar{p}} A_{j}(X, d)$ is a convex metric space, and $\bigcap_{j \in \bar{p}} A_{j}$ is nonempty and convex. Then, from the convexity of $\bigcap_{j \in \bar{p}} A_{j}$ and (25), one gets that $\left[\bar{x}_{1}^{(n+1)}, \bar{x}_{2}\right] \subset\left[\bar{x}_{1}^{(n)}, \bar{x}_{2}\right] \subset \bigcap_{j \in \bar{p}} A_{j}, \bar{x}_{1}^{(n)}=\bar{x}_{2}$ and $\bar{x}_{1}^{(n)} \preceq \bar{x}_{2} ; \forall n \in \mathbf{Z}_{0+}$ and then there are infinitely many fixed points of $T: \bigcup_{i \in \bar{p}} A_{i} \rightarrow \bigcup_{i \in \bar{p}} A_{i}$ so that $T \bar{x}_{1}$ has infinite cardinal and $T: \bigcup_{i \in \bar{p}} A_{i} \rightarrow \bigcup_{i \in \bar{p}} A_{i}$ would not be a multivalued self-mapping, a contradiction. Thus, $\bar{x}_{1}=\bar{x}_{2}=$ $\bar{x}$ is the unique fixed point of $T: \bigcup_{i \in \bar{p}} A_{i} \rightarrow \bigcup_{i \in \bar{p}} A_{i}$ and also the unique fixed point of $T^{p}: \bigcup_{i \in \bar{p}} A_{i} \rightarrow \bigcup_{i \in \bar{p}} A_{i}$ using a similar argument to the above one to prove the uniqueness. Hence, property (iii) follows.

The proof of property (iv) follows directly from the above properties (i)-(iii) and property (iii) of Theorem 1; property (v) follows directly, since $A_{i} \subset X ; \forall i \in \bar{p}$ being closed implies that $\bigcap_{i \in \bar{p}} A_{i}$ is also closed, which together with the condition that $\bigcap_{i \in \bar{p}} A_{i}$ is convex, leads to the property that $\left(\bigcap_{i \in \bar{p}} A_{i}, d\right)$ is a convex metric space if $X$ is an Euclidean vector space while the complementary to $\bigcap_{i \in \bar{p}} A_{i}$ in $X$ is not invoked in the proof of the uniqueness of the fixed point so that if $\left(\bigcap_{i \in \bar{p}} A_{i}, d\right)$ is a convex metric space the uniqueness proof follows as that of property $(\mathrm{v})$.

Remarks 1. (1) Note that the restricted composite multivalued self-mapping $\widehat{T}_{j}: A_{j} \rightarrow A_{j} ; \forall j \in \bar{p}$ can be extended in a natural way to the composite self-mapping $T^{p}: \bigcup_{i \in p} A_{i} \rightarrow$ $\bigcup_{i \in p} A_{i}$ in the sense that $T^{p} \mid A_{j}=\widehat{T}_{j} ; \forall j \in \bar{p}$.

(2) The convexity of the subsets $A_{i} ; i \in \bar{p}$ is not required in Theorem 2(iii) but that of their intersection.

(3) Finally, note that a convex set in a Euclidean space is convex metric space under the Euclidean induced norm and that closed subsets of Euclidean spaces are convex metric spaces if and only if they are convex. This property is used in the proof of property (v) of Theorem 2. Finally, note that Theorem 2(v) holds independently of the metric (not necessarily for a norm-induced metric) and that properties (iii)-(iv) do not require that the subsets $A_{i} \subset X$ for $i \in \bar{p}$ are convex but that their intersection is convex.

\section{The Main Result on Best Proximity Points for Nonintersecting Subsets}

An "ad hoc" version of Theorem 2 will be obtained in this section for the case of nonintersecting subsets by proving the convergence to unique best proximity points within each subset $A_{i}$, which are also unique fixed points of each of the composed self-mappings $\widehat{T}_{i}: A_{i} \rightarrow A_{i} ; \forall i \in \bar{p}$. It is assumed that $(X,\|\|)$ is a uniformly convex Banach space endowed with the partial order $\preceq$ and that the subsets $A_{i}$; $\forall i \in \bar{p}$ are nonempty, closed, and convex sets. The following remark describes conditions to characterize a class of Banach spaces from complete metric spaces provided that the norm is induced from a metric.

Remark 3. It is well known that a norm defines a metric. In this sense, a Banach space $(X,\|\|)$ can be considered also a complete metric space $(X, d)$ under the norm-induced metric. To practical effects, the induced metric is identical to the norm. The contrary is not true in general since metrics are subject to less restrictive conditions than norms. However, under certain conditions, as for instance, if the metric is homogeneous and translation-invariant, then it can be considered as a norm in a natural way, say, a metricinduced norm. In this case, we can also consider the norm 
to be identical to the metric-induced norm. If $(X, d)$ is a complete metric space and $X$ is a vector space and $d: X \times$ $X \rightarrow \mathbf{R}_{0+}$ is a homogeneous and translation invariant metric, then $(X,\|\|)$ is also a Banach space under such a metricinduced norm $d: X \times X \rightarrow \mathbf{R}_{0+}$.

The next result is an "ad hoc" version for this paper of previous technical results. See Lemma 3.7, Lemma 3.8 and Theorem 3.10 in [33].

Lemma 4 (Lemma 3.7 and Lemma 3.8 of [33]). Let $A_{i} \subseteq X$ for $i \in \bar{p}$ be nonempty closed subsets of the vector space $X$ of a uniformly convex Banach space $(X,\|\|)$ with norm-induced metric $d: X \times X \rightarrow \mathbf{R}_{0+}$ and $d\left(A_{i}, A_{i+1}\right)=D_{i}$ and either $A_{i}$ or $A_{i+1}$ for $i \in \bar{p}$ are, furthermore, convex (i.e., at least one of each two adjacent subsets is, in addition, convex). Consider sequences $\left\{x_{n p+i}\right\}_{n \in \mathbf{Z}_{0+}} \subset A_{i}$ and $\left\{z_{n p+i}\right\}_{n \in \mathbf{Z}_{0+}} \subset A_{i}$ and $\left\{y_{n p+i+1}\right\}_{n \in \mathbf{Z}_{0+}} \subset A_{i+1}$ satisfying

(1) $\left\|z_{n p+i}-y_{n p+i}\right\| \rightarrow D_{i}$ as $n \rightarrow \infty$ for any given $i \in \bar{p}$.

(2) For every $\varepsilon \in \mathbf{R}_{+}$, there is $n_{0} \in \mathbf{Z}_{0+}$ such that $\| x_{m p+i}-$ $y_{n p+i+1} \| \leq D+\varepsilon$ for all $m, n \in \mathbf{Z}_{0+}$ with $m>n \geq n_{0}$ for any given $i \in \bar{p}$.

Then, the following properties hold:

(i) For every $\varepsilon \in \mathbf{R}_{+}$, there is $n_{1} \in \mathbf{Z}_{0+}$ such that $\| x_{m p+i}-$ $z_{n p+i} \| \leq \varepsilon$ for all $m, n \in \mathbf{Z}_{0+}$ with $m>n \geq n_{1}$ for any given $i \in \bar{p}$.

(ii) If $\left\|z_{n p+i}-y_{n p+i}\right\| \rightarrow D_{i}$ as $n \rightarrow \infty$ and $\| x_{n p+i}-$ $y_{n p+i} \| \rightarrow D_{i}$ as $n \rightarrow \infty$ then $\left\|x_{n p+i}-z_{n p+i}\right\| \rightarrow 0$ as $n \rightarrow \infty$ for any given $i \in \bar{p}$.

(iii) $\left\{x_{n p+i+j}\right\}_{n \in \mathbf{Z}_{0+}}$ and $\left\{z_{n p+i+j}\right\}_{n \in \mathbf{Z}_{0+}}$ are Cauchy sequences; $\forall j \in \overline{p-1} \cup\{0\}$ and the given $i \in \bar{p}$.

The proof of Lemma 4(i)-(ii) is supported by the nonemptiness, closeness, and convexity of the subsets $A_{j} \subseteq$ $X ; j \in \bar{p}$ and the uniform convexity of the Banach space $(X,\|\|)[33]$. The following main result for multivalued $p$-cyclic self-mappings is obtained from Theorem 1 and Lemma 4 while taking into account Remark 3.

Theorem 5. Let $T: \bigcup_{i \in \bar{p}} A_{i} \rightarrow \bigcup_{i \in \bar{p}} A_{i}$ be a multivalued $p(\geq 2)$-cyclic self-mapping on $\bigcup_{i \in \bar{p}} A_{i}$ with $A_{i} \in C B(X) \subseteq X$; $\forall i \in \bar{p}$ being all nonempty and convex with $D_{i}=d\left(A_{i}, A_{i+1}\right)$; $\forall i \in \bar{p}$. Assume the following:

(1) Let $X$ be a vector space and let $(X, d)$ be a convex complete metric space with $d: X \times X \rightarrow \mathbf{R}_{0+}$ being a homogeneous translation-invariant metric which induces a norm \|| $\|$ on $X$ such that $(X,\|\|)$ is a Banach space.

(2) $(X,\|\|)$ is a uniformly convex Banach space with metric convexity.

(3) The complete metric space $(X, d)$, equivalently, the Banach space $(X,\|\|)$, is endowed with a partial order $\preceq$ defined by (5) with $x=x_{i}\left(\in A_{i}\right) \preceq y \in T x\left(\subseteq A_{i+1}\right)$ for any $(x, y) \in A_{i} \times A_{i+1}$ and some given $i \in \bar{p}$ such that the resulting $(X, \preceq)$ partially ordered space is subject to assumptions (1)-(4) of Theorem 1 and assumption (7) of Theorem 2.

Then, the following properties hold.

(i) There are unique best proximity points $\bar{x}_{j+1} \in T \bar{x}_{j} \subseteq$ $A_{j+1}$ with $d\left(\bar{x}_{j}, \bar{x}_{j+1}\right)=d\left(\bar{x}_{j}, T \bar{x}_{j+1}\right)=D_{j}$, for each $j \in \bar{p}$ which are also unique fixed points of each of the restricted composite self-mappings $\widehat{T}_{j}\left(\equiv T^{p} \mid A_{j}\right)$ : $A_{j} \rightarrow A_{j} ; \forall j \in \bar{p}$.

(ii) Take any $x=x_{i}\left(\in A_{i}\right) \preceq y=x_{i+1} \in T x_{i}$ for any given $i \in \bar{p}$ (i.e., $x$ and $y$ are partially ordered with respect to the partial ordered set $(X, \preceq)$ and consider the partially ordered sequences $\left\{x_{n p+j}\right\}$, being nondecreasing with respect to $\preceq$ while satisfying $x_{n p+j+1} \in T x_{n p+j} ; \forall j \in \bar{p}$ of first element subject to $x=x_{i}\left(\epsilon A_{i}\right) \preceq y=x_{i+1} \epsilon$ $T x_{i}$ for any given $i \in \bar{p}$. Then, each of such sequences $\left\{x_{n p+j}\right\}$ converges to the unique best proximity point $\bar{x}_{j}$ in $A_{j} ; \forall j \in \bar{p}$ which is also the unique fixed point of each of the restricted composite self-mapping $\widehat{T}_{j}$ : $A_{j} \rightarrow A_{j}$. If $\bigcap_{i \in \bar{p}} A_{i} \neq \varnothing$, then $\bar{x}=\bar{x}_{j} \in \bigcap_{i \in \bar{p}} A_{i}$ is the unique fixed point of $T: \bigcup_{i \in \bar{p}} A_{i} \rightarrow \bigcup_{i \in \bar{p}} A_{i}$, $\widehat{T}_{j}\left(\equiv T^{p} \mid A_{j}\right)$ and a fixed point of $T^{p}: \bigcup_{i \in \bar{p}} A_{i} \rightarrow$ $\bigcup_{i \in \bar{p}} A_{i} ; \forall j \in \bar{p}$.

(iii) If assumption (4) of Theorem 1 is replaced by its assumption (5), then the convergence to the above unique best proximity points holds for partially ordered sequences of first element $x \in \bigcup_{i \in \bar{p}} A_{i}$.

Proof. Note from the various hypothesis the uniformly convex Banach space $(X,\|\|)$ possesses the metric convexity property with respect to the norm metric \|\| while it is endowed with a partial order $\preceq$ under assumptions (1)-(4) of Theorem 1. From property (ii) of Theorem 1, (8), the nonemptiness and closeness of the subsets $A_{i} \subseteq X ; \forall i \in \bar{p}$, and Lemma 4(i)-(ii), it follows that

$$
\begin{array}{r}
\exists \lim _{n \rightarrow \infty} d\left(x_{n p+i+j+1}, x_{n p+i+j}\right)=D_{i+j} ; \\
\exists \lim _{n \rightarrow \infty} d\left(x_{(n+1) p+i+j}, x_{n p+i+j}\right)=0 ; \\
\forall j \in \overline{p-1} \cup\{0\},
\end{array}
$$

where $x_{n p+i+j} \in T x_{n p+i+j-1} \subseteq A_{i+j}, x_{(n+1) p+i+j} \in$ $T^{p} x_{n p+i+j-1} \subseteq T x_{(n+1) p+i+j-1} \subseteq A_{i+j} ; \forall j \in \overline{p-1} \cup\{0\}$, $\forall n \in \mathbf{Z}_{0+}$ for the given $i \in \bar{p}$ and the iterated sequences; $\left\{x_{n p+i+j}\right\}_{n \in \mathbf{Z}_{0+}} ; \forall j \in \overline{p-1} \cup\{0\}$ and the given $i \in \bar{p}$ are partially ordered with respect to the partial order $\preceq$, from Theorem 1, of first element $x_{i+j}=x_{j}$ generated from the iteration $x_{n p+i+j} \in$ $T x_{n p+i+j-1} ; \forall j \in \overline{p-1} \cup\{0\}$ and the given $i \in \bar{p}$ are all Cauchy sequences. Since $(X, d) \equiv(X,\|\|)$ is complete, it follows that $x_{n p+i+j} \rightarrow \bar{x}_{i+j}\left(\in T^{p} x_{(n-1) p+i+j} \subseteq T x_{n p+i+j-1} \subseteq A_{i+j}\right)$ and $x_{n p+i+j+1} \rightarrow \bar{x}_{i+j+1}\left(\in T^{p} x_{(n-1) p+i+j+1} \subseteq T x_{n p+i+j} \subseteq A_{i+j+1}\right)$ as $n \rightarrow \infty ; \forall j \in \overline{p-1} \cup\{0\}$ and the given $i \in \bar{p}$ since $A_{j} \subseteq X$ 
is nonempty, bounded and closed; $\forall j \in \bar{p}$ and the given $i \in \bar{p}$. Thus, one gets from (26), since $A_{j} \subseteq X$ is nonempty, bounded and closed, and then boundedly compact, and also approximatively compact with respect to $A_{j-1}[8,35]$, that:

$$
\begin{aligned}
D_{i+j} & \leq d\left(x_{n p+i+j+1}, x_{n p+i+j}\right) \\
& \longrightarrow d\left(\bar{x}_{i+j}, \bar{x}_{i+j+1}\right)=D_{i+j} \\
& =d\left(\bar{x}_{i+j}, T \bar{x}_{i+j}\right) \text { as } n \longrightarrow \infty ;
\end{aligned}
$$

$\forall j \in \overline{p-1} \cup\{0\}$ and the given $i \in \bar{p}$, where $\bar{x}_{i+j+1} \in$ $T^{p} \bar{x}_{i+j+1-p} \subseteq T \bar{x}_{i+j} ; \forall j \in \overline{p-1} \cup\{0\}$ and the given $i \in \bar{p}$. Since all the subsets $A_{j} \subset X ; \forall j \in \bar{p}$ are nonempty, closed, and boundedly compact; $\forall j \in \bar{p}$ then $\bar{x}_{j} \in A_{j}$ is a best proximity point in $A_{j}$ of $T: \bigcup_{i \in \bar{p}} A_{i} \rightarrow \bigcup_{i \in \bar{p}} A_{i}$ and it is also a fixed point of the restricted composite self-mapping $\widehat{T}_{j}: \bigcup_{i \in \bar{p}} A_{i}\left|A_{j} \rightarrow \bigcup_{i \in \bar{p}} A_{i}\right| A_{j} ; \forall j \in \bar{p}$. Thus, there are Cauchy, then convergent since $(X, d)$ is complete, sequences $\left\{x_{n p+i+j}\right\}_{n \in \mathbf{Z}_{0+}}$ with respective first elements $x_{i+j} \in T x_{i+j-1}$; $\forall j \in \overline{p-1} \cup\{0\}$ and the given $i \in \bar{p}$, each being convergent to $\bar{x}_{i+j} \in A_{i+j}$, such that $x=x_{i}$ is the first element of $\left\{x_{n p+i}\right\}_{n \in \mathbf{Z}_{0+}} \subseteq A_{i}$ which consists of partially ordered elements with respect to the partial order $\preceq$ such that

$$
\begin{aligned}
x_{i+j} & \preceq \cdots \preceq x_{(n+1) p+i+j}\left(\in T^{p} x_{n p+i+j} \subseteq T x_{(n+1) p+i+j-1}\right) \\
& \preceq x_{(n+1) p+i+j} \preceq \cdots \preceq \bar{x}_{i+j} \\
& =\lim _{n \rightarrow \infty} x_{n p+i+j}
\end{aligned}
$$

with $\left\{x_{\ell p+i+j}\right\} \subseteq A_{i+j} ; j \in \overline{p-1} \cup\{0\}, \forall \ell \in \mathbf{Z}_{0+}$ for the given $i \in \bar{p}$. But $\bar{x}_{i+j} \in A_{i+j} ; \forall j \in \overline{p-1} \cup\{0\}$ and the given $i \in \bar{p}$, is a fixed point of the restricted composite self-mapping $\widehat{T}_{j}: \bigcup_{i \in \bar{p}} A_{i}\left|A_{j} \rightarrow \bigcup_{i \in \bar{p}} A_{i}\right| A_{j}, \forall j \in \bar{p}$ and a fixed point of the composite self-mapping $T^{p}: \bigcup_{i \in \bar{p}} A_{i} \rightarrow \bigcup_{i \in \bar{p}} A_{i}$ from Lemma 4(iii) to which the partially ordered sequences of first element $x=x_{i} \in A_{i}$ converge. It is also a best proximity point in $A_{i+j}$ of the self-mapping $T: \bigcup_{i \in \bar{p}} A_{i} \rightarrow \bigcup_{i \in \bar{p}} A_{i}$ from Lemma 4(iii) and the second part of Lemma 4(ii). Then, $\bar{x}_{j} \in T \bar{x}_{j} \subseteq T^{p} \bar{x}_{j} ; \forall j \in \bar{p}$. The uniqueness property of each of those $p$ best proximity points $\bar{x}_{j} \in T \bar{x}_{j}$ in each of the subsets $A_{j} \subseteq X$ follows from their uniqueness as fixed points of the restricted self-mappings $\widehat{T}_{j}: \bigcup_{i \in \bar{p}} A_{i} \mid A_{j} \rightarrow$ $\bigcup_{i \in \bar{p}} A_{i} \mid A_{j}$ from Theorem 2 , since $(X, d)$ is a convex metric space and the subsets $A_{j} \subseteq X$ are convex; $\forall j \in \bar{p}$. On the other hand, it turns out that if all the subsets have nonempty intersection, such an intersection is convex so that the best proximity points are all identical and the unique fixed point of $T: \bigcup_{i \in \bar{p}} A_{i} \rightarrow \bigcup_{i \in \bar{p}} A_{i}$ and $T^{p}: \bigcup_{i \in \bar{p}} A_{i} \rightarrow \bigcup_{i \in \bar{p}} A_{i}$ from Theorem 2, this leads to the proofs of properties (i)-(iii).

Remarks 2. (1) Theorem 5 proves the uniqueness of the best proximity points for any partially ordered sequences with first elements in any of the subsets of the multivalued $p$-cyclic self-mapping on $\bigcup_{i \in \bar{p}} A_{i}$ satisfying assumptions (1)-(4) of
Theorem 1 as it was commented, in Section 2 concerning such a theorem, the given $A_{i} \subset X$ for some $i \in \bar{p}$ to select the first two elements of the partial order can be chosen arbitrarily by construction from (6), namely, from assumption (4) of Theorem 1.

(2) The value of the individual contractive constants being less than, equal to, or larger than one for each pair of adjacent subsets is irrelevant in Theorem 5 provided that its product is less than one. Note also that Theorem 5 holds also if the distances between each pair of adjacent subsets are not necessarily identical.

(3) Note also that, for Euclidean metric, the convexity of $X$ is kept as hypothesis for the uniqueness of the best proximity points of the multivalued self-mapping, since although the subsets $A_{i}$ of $X, i \in \bar{p}$ are convex, the existence of points belonging to such subsets guaranteeing the equality in the triangle inequality for the metric would not be otherwise guaranteed, since such sets are disjoint and pair-wise disjoint.

(4) It can be observed that the metric convexity of the space $(X, d)$ cannot be relaxed to that of $\left(\bigcap_{i \in \bar{p}} A_{i}, d\right)$, since the subsets $A_{i} \subset X$ do not necessarily intersect.

(5) Note that the results of Sections 2 and 3 obtained from the contractive condition (5) also hold for multivalued selfmappings $T: \bigcup_{i \in \bar{p}} A_{i} \rightarrow \bigcup_{i \in \bar{p}} A_{i}$ which are not cyclic; that is, $T\left(A_{i}\right) \cap \bar{A}_{i+1} \neq \varnothing$ for some $i \in \bar{p}$ but fulfil the condition $T x \cap A_{i+1} \neq \varnothing ; \forall x \in A_{i}, \forall i \in \bar{p}$.

\section{Example}

Consider two bounded and closed real subsets $A_{1}=$ $[\varepsilon, M] ; A_{2}=[-M,-\varepsilon]=-A_{1}$ for nonnegative positive real constants $\varepsilon$, $M$ with $\varepsilon \leq M$ under the Euclidean metric so that $D=d\left(A_{1}, A_{2}\right)=2 \varepsilon$.

Consider also a scalar discrete dynamic system of state $x_{k}$ operating at each state value under $N$ tentative feedback controls $u_{k}^{\left(i_{k}\right)}=-K_{k}^{\left(i_{k}\right)} x_{k}^{\left(i_{k}\right)} ; \forall i_{k} \in I_{k}$, where the indexing set of tentative states at the $(k+1)$-th sampling point is defined by

$$
\begin{aligned}
I_{k+1} & =I_{k} \times \bar{N} \\
& =I_{0} \times \bar{N} \times \cdots \times \bar{N} \\
& =\{1\} \times \bar{N}^{k}, \quad \forall k \in \mathbf{Z}_{0+},
\end{aligned}
$$

where " $x$ " stands for the Cartesian product of sets, $x_{0}^{\left(i_{0}\right)}=$ $x_{0}^{(1)}=x \in[-M,-\varepsilon] \cup[\varepsilon, M]$ is the initial point of an iteration through a self-mapping $T$ from $[-M,-\varepsilon] \cup[\varepsilon, M]$ to itself and $I_{0}=\{1\}$. Then the discrete state trajectory takes values in alternated points at $A_{1}$ and $A_{2}$ from the initial state condition $x$ such that $x_{k+1}^{\left(i_{k+1}\right)} \in T x_{k}^{\left(i_{k}\right)}=\left\{x_{k+1}^{\left(i_{k}, 1\right)}, \ldots, x_{k+1}^{\left(i_{k}, N\right)}\right\} ; \forall k \in \mathbf{Z}_{0+}$ obtained as follows:

$$
\begin{aligned}
& x_{k+1}^{\left(i_{k+1}\right)} \\
& = \begin{cases}\bar{x}_{k+1}^{\left(i_{k+1}\right)} & \text { if }\left|\bar{x}_{k+1}^{\left(i_{k+1}\right)}\right| \in[\varepsilon, M], \\
M \operatorname{sgn} \bar{x}_{k+1}^{\left(i_{k+1}\right)} & \text { if }\left|\bar{x}_{k+1}^{\left(i_{k+1}\right)}\right|>M, \forall i_{k} \in I_{k}, \forall k \in \mathbf{Z}_{0+}, \\
\varepsilon \operatorname{sgn} \bar{x}_{k+1}^{\left(i_{k+1}\right)} & \text { if }\left|\bar{x}_{k+1}^{\left(i_{k+1}\right)}\right|<\varepsilon,\end{cases}
\end{aligned}
$$


where

$$
\begin{aligned}
\bar{x}_{k+1}^{\left(i_{k+1}\right)} & =a_{k} x_{k}^{\left(i_{k}\right)}+b_{k} u_{k}^{\left(i_{k}\right)} \\
& =\left(a_{k}-b_{k} K_{k}^{\left(i_{k}\right)}\right) x_{k}^{\left(i_{k}\right)} ; \quad \forall i_{k} \in I_{k}, \forall k \in \mathbf{Z}_{0+}
\end{aligned}
$$

with $a_{k} \neq 0$ and $b_{k}>0$ being nonzero real numbers under a sequence of controllers of gains

$$
K_{k}^{\left(i_{k}\right)}=\frac{\rho_{k}^{\left(i_{k}\right)}+a_{k}}{b_{k}}, \quad \rho_{k}^{\left(i_{k}\right)} \in[0, \rho] ; \forall i_{k} \in I_{k}, \forall k \in \mathbf{Z}_{0+}
$$

for some nonnegative real constant $\rho$ so that, after replacing (32) into (31), this leads to the controlled closed-loop trajectory sequence given by (30) subject to $\bar{x}_{k+1}^{\left(i_{k+1}\right)}=-\rho_{k}^{\left(i_{k}\right)} x_{k}^{\left(i_{k}\right)}$; $\forall i_{k} \in I_{k}$ with $\left|x_{k+1}^{\left(i_{k+1}\right)}\right| \leq \min \left(\max \left(\rho_{k}^{\left(i_{k}\right)}\left|x_{k}^{\left(i_{k}\right)}\right|, \varepsilon\right), M\right) ; \forall i_{k} \in I_{k}$, $\forall k \in \mathbf{Z}_{0+}$. Assume that (5) holds for $x \preceq y$ so that, for some $y \in T x$ and some $z \in T y$, one gets

$$
d(z, y) \leq H(T x, T y) \leq K d(x, y)+2(1-K) \varepsilon
$$

for some $K \in(0,1)$. Note also from (30)-(31) that (5) is guaranteed under the set of constraints below:

$$
\begin{aligned}
2 \varepsilon & \leq d\left(x_{k+2}^{\left(i_{k+1}\right)}, x_{k+1}^{\left(i_{k}\right)}\right) \\
& \leq d\left(\bar{x}_{k+2}^{\left(i_{k+1}\right)}, x_{k+1}^{\left(i_{k}\right)}\right) \\
& =\left|\bar{x}_{k+2}^{\left(i_{k+1}\right)}-x_{k+1}^{\left(i_{k}\right)}\right|=\left|\bar{x}_{k+2}^{\left(i_{k+1}\right)}\right|+\left|x_{k+1}^{\left(i_{k}\right)}\right| \\
& =\left|a_{k+1}-b_{k+1} K_{k+1}^{\left(i_{k+1}\right)}-1\right|\left|x_{k+1}^{\left(i_{k+1}\right)}\right| \\
& \leq\left|a_{k+1}-b_{k+1} K_{k+1}^{\left(i_{k+1}\right)}-1\right| \min \left(\left|\bar{x}_{k+1}^{\left(i_{k+1}\right)}\right|, M\right) \\
& \leq\left|a_{k+1}-b_{k+1} K_{k+1}^{\left(i_{k+1}\right)}-1\right| \min \left(\rho_{k}^{\left(i_{k}\right)}\left|x_{k}^{\left(i_{k}\right)}\right|, M\right) \\
& \leq \min \left(d_{0}, K d\left(x_{k+1}^{\left(i_{k+1}\right)}, x_{k}^{\left(i_{k}\right)}\right)+2(1-K) \varepsilon\right) \\
& =\min \left(d_{0}, K\left|a_{k}-b_{k} K_{k}^{\left(i_{k}\right)}-1\right|\left|x_{k}^{\left(i_{k}\right)}\right|+2(1-K) \varepsilon\right) \\
& \leq \min \left(d_{0}, K\left|a_{k}-b_{k} K_{k}^{\left(i_{k}\right)}-1\right| M+2(1-K) \varepsilon\right) \\
& \leq d_{0} ; \quad \text { for some } i_{k} \in I_{k}, \forall k \in \mathbf{Z}_{0+}
\end{aligned}
$$

which hold provided that there is at least a controller gain sequence $\left\{K_{k}^{\left(i_{k}\right)}\right\}_{i_{k} \in I_{k}}$ subject to the constraints:

$$
\begin{gathered}
d_{0}>\max \left(2 \varepsilon, M\left(a_{k}+\left|a_{k}-1\right|\right)\right), \\
\min \left(1, \frac{d_{0}}{M}-a_{k}-\left|a_{k}-1\right|, \frac{K\left|a_{k+1}-b_{k} K_{k}^{\left(i_{k}\right)}-1\right|+\left(2 \varepsilon(1-K) /\left|x_{k}\right|\right)}{\left|a_{k+2}-b_{k+1} K_{k+1}^{\left(i_{k+1}\right)}-1\right|}\right) \\
\min \left(1, \frac{d_{0}}{M}-a_{k}-\left|a_{k}-1\right|, \frac{K\left|a_{k+1}-b_{k} K_{k}^{\left(i_{k}\right)}-1\right|}{\left|a_{k+2}-b_{k+1} K_{k+1}^{\left(i_{k+1}\right)}-1\right|}\right) \\
\text { if } \varepsilon=0
\end{gathered}
$$

for some $i_{k} \in I_{k} ; \forall k \in \mathbf{Z}_{0+}$. The above constraints guarantee the generation of an iterated nondecreasing partially ordered sequence $S(x)=\left\{x=x_{0}^{(1)}, x_{1}^{\left(i_{i}\right)}, \ldots, x_{n}^{\left(i_{n}\right)}, \ldots\right\}$ by the selfmapping $T$ on $[-M,-\varepsilon] \cup[\varepsilon, M]$ with respect to the partial order " $\leq$ " under a sequence of control gains satisfying $0<$ $K_{k}^{\left(i_{k}\right)}=\left(\rho_{k}^{\left(i_{k}\right)}+a_{k}\right) / b_{k} \leq\left(1 /\left|b_{k}\right|\right)\left(\left(d_{0} / M\right)-\left|a_{k}-1\right|\right)$ and $\left|a_{k}-1\right|<2 \varepsilon / M \leq d_{0} / M ; \forall k \in \mathbf{Z}_{0+}$.

It follows from Theorem 5 that such a sequence has two subsequences, built by keeping the same order with alternated terms of $S(x)$, converging, respectively, to the unique best proximity points, $x_{1}^{*}=\varepsilon \in A_{1}$ and $x_{2}^{*}=-\varepsilon \in A_{2}$ of the selfmapping. If $\varepsilon=0$ both such sequences and $S(x)$ converge to the resulting unique fixed point of the 2-cyclic composite selfmapping $T$ on $[-M,-\varepsilon] \cup[\varepsilon, M]$. If the considered subsets are $A_{1}=[-\varepsilon, M] ; A_{2}=[-M, \varepsilon]=-A_{1}$, the first term of the chain of inequalities (34) is valid with the replacement $2 \varepsilon \rightarrow 0$ while the terms $2(1-K) \varepsilon$ are all zeroed in such a chain of inequalities. Thus, (34) holds if there is a sequence of controller gains satisfying

$$
\begin{aligned}
0 & <K_{k}^{\left(i_{k}\right)} \\
& =\frac{\rho_{k}^{\left(i_{k}\right)}+a_{k}}{b_{k}} \\
& \leq \frac{1}{\left|b_{k}\right|}\left(\frac{d_{0}}{K M}-\left|a_{k}-1\right|\right) \quad \text { for some } i_{k} \in I_{k} ; \forall k \in \mathbf{Z}_{0+}
\end{aligned}
$$

under sufficiency-type constraints $d_{0}>K M$ if $\left|a_{k}\right|<$ $d_{0} / K M-1 ; \forall k \in \mathbf{Z}_{0+}$ or $d_{0}<K M$ and $a_{k}>1-d_{0} / K M$ if $a_{k} \epsilon$ $[0,1] ; \forall k \in \mathbf{Z}_{0+}$. Thus, $S(x)=\left\{x=x_{0}^{(1)}, x_{1}^{\left(i_{i}\right)}, \ldots, x_{n}^{\left(i_{n}\right)}, \ldots\right\}$ converges to $x^{*}=0$ which is the a unique fixed point of $T$ on $[-M,-\varepsilon] \cup[\varepsilon, M]$. 
The following extension of the example is direct. Assume that (31) is replaced by the second-order uncoupled discrete dynamic system:

$$
\begin{aligned}
& \bar{x}_{k+1}^{\left(i_{k+1}\right)}=\left(\bar{x}_{1 k+1}^{\left(i_{k+1}\right)}, \bar{x}_{2 k+1}^{\left(i_{k+1}\right)}\right)^{T} \\
& =A_{k} x_{k}^{\left(i_{k}\right)}+B_{k} u_{k}^{\left(i_{k}\right)} \\
& =\left(A_{k}-B_{k} K_{k}^{\left(i_{k}\right)^{T}}\right) x_{k}^{\left(i_{k}\right)} ; \quad \forall i_{k} \in I_{k}, \forall k \in \mathbf{Z}_{0+},
\end{aligned}
$$

where the superscript $T$ stands for transposition and $x_{k+1}^{\left(i_{k+1}\right)}=$ $\left(x_{1 k+1}^{\left(i_{k+1}\right)}, x_{2 k+1}^{\left(i_{k+1}\right)}\right)^{T}$, subject to the tentative scalar feedback controls $u_{k}^{\left(i_{k}\right)}=-K_{k}^{\left(i_{k}\right)^{T}} x_{k}^{\left(i_{k}\right)}$, where $A_{k}=\operatorname{Diag}\left(a_{1 k}, a_{2 k}\right)$ is a sequence of real $2 \times 2$ diagonal matrices, $B_{k}=\left(b_{1 k}, b_{2 k}\right)^{T}$ is a sequence of two dimensional real column vectors, $K_{k}^{\left(i_{k}\right)^{T}}$ are sequences of two-dimensional real row vectors. The set of inequalities (34) are replaced by parallel ones by using the maxima for norms/distances of the two state vector components. The resulting inequalities are obtained from the initial basic constraints:

$$
\begin{aligned}
2 \varepsilon & \leq d\left(x_{k+2}^{\left(i_{k+1}\right)}, x_{k+1}^{\left(i_{k}\right)}\right) \\
& \leq d\left(\bar{x}_{k+2}^{\left(i_{k+1}\right)}, x_{k+1}^{\left(i_{k}\right)}\right) \\
& =\max _{1 \leq j \leq 2} d\left(\bar{x}_{j k+2}^{\left(i_{k+1}\right)}, x_{j, k+1}^{\left(i_{k}\right)}\right)
\end{aligned}
$$

for some $i_{k} \in I_{k}, \forall k \in \mathbf{Z}_{0+}$. To keep the validity of (30) for each state component, the constraints (35a) and (35b) are replaced by

$$
\begin{gathered}
d_{0}>\max _{1 \leq j \leq 2} \max \left(2 \varepsilon, M\left(a_{j k}+\left|a_{j k}-1\right|\right)\right), \\
\min \left(1, \min _{1 \leq j \leq 2}\left(\frac{d_{0}}{M}-a_{j k}-\left|a_{j k}-1\right|\right), \min _{1 \leq j \leq 2} \frac{K\left|a_{j, k+1}-b_{j k} K_{j k}^{\left(i_{k}\right)}-1\right|+\left(2 \varepsilon(1-K) /\left|x_{j k}\right|\right)}{\left|a_{j, k+2}-b_{j, k+1} K_{j, k+1}^{\left(i_{k+1}\right)}-1\right|}\right) \\
\min \left(1, \min _{1 \leq j \leq 2}\left(\frac{d_{0}}{M}-a_{j k}-\left|a_{j k}-1\right|\right), \min _{1 \leq j \leq 2} \frac{K\left|a_{j, k+1}-b_{j k} K_{j k}^{\left(i_{k}\right)}-1\right|}{\left|a_{j, k+2}-b_{j, k+1} K_{j, k+1}^{\left(i_{k+1}\right)}-1\right|}\right)
\end{gathered}
$$

for some $i_{k} \in I_{k} ; \forall k \in \mathbf{Z}_{0+}$.

\section{Acknowledgments}

The author is very grateful to the Spanish Government for its support of this research through Grant DPI2012-30651 and to the Basque Government by its support of this research through Grants IT378-10 and SAIOTEK S-PE12UN015. He is also grateful to UPV/EHU for its financial support through UFI 2011/07 and to the referees for their useful comments.

\section{References}

[1] Y. Yao, M. A. Noor, Y.-C. Liou, and S. M. Kang, "Iterative algorithms for general multivalued variational inequalities," Abstract and Applied Analysis, vol. 2012, Article ID 768272, 10 pages, 2012.

[2] M. De la Sen, "Stable iteration procedures in metric spaces which generalize a Picard-type iteration," Fixed Point Theory and Applications, vol. 2010, Article ID 953091, 15 pages, 2010.

[3] M. Ratchagit and K. Ratchagit, "Asymptotic stability and stabilization of fixed points for iterative sequence," International Journal of Research and Reviews in Computer Science, vol. 2, no. 4, pp. 987-989, 2011.

[4] D. Doric and R. Lazović, "Some Suzuki-type fixed point theorems for generalized multivalued mappings and applications," Fixed Point Theory and Applications, vol. 2011, p. 40, 2011.
[5] L. J. Ciric, "Multi-valued nonlinear contraction mappings," Nonlinear Analysis. Theory, Methods \& Applications A, vol. 71, no. 7-8, pp. 2716-2723, 2009.

[6] L. J. Ciric, "Fixed points for generalized multi-valued contractions," Matematicki Vesnik, vol. 9, no. 24, pp. 265-272, 1972.

[7] S. L. Singh, S. N. Mishra, and S. Jain, "Round-off stability for multi-valued maps," Fixed Point Theory and Applications, vol. 2012, p. 12, 2012.

[8] S. L. Singh, S. N. Mishra, R. Chugh, and R. Kamal, "General common fixed point theorems and applications," Journal of Applied Mathematics, vol. 2012, Article ID 902312, 14 pages, 2012.

[9] W. Laowang and B. Panyanak, "Common fixed points for some generalized multivalued nonexpansive mappings in uniformly convex metric spaces," Fixed Point Theory and Applications, p. 201120, 2011.

[10] H. Khandani, S. M. Vaezpour, and B. Sims, "Common fixed points of generalized multivalued contraction on complete metric spaces," Journal of Computational Analysis and Applications, vol. 13, no. 6, pp. 1025-1038, 2011.

[11] M. Abbas, "Coincidence points of multivalued $f$-almost nonexpansive mappings," Fixed Point Theory, vol. 13, no. 1, pp. 3-10, 2012.

[12] Sh. Rezapour and P. Amiri, "Fixed point of multivalued operators on ordered generalized metric spaces," Fixed Point Theory, vol. 13, no. 1, pp. 173-178, 2012.

[13] A. Petrusel and G. Petrusel, "Multivalued Picard operators," Journal of Nonlinear and Convex Analysis, vol. 13, no. 1, pp. 157171, 2012. 
[14] T. P. Petru, A. Petruşel, and J.-C. Yao, "Ulam-Hyers stability for operatorial equations and inclusions via nonself operators," Taiwanese Journal of Mathematics, vol. 15, no. 5, pp. 2195-2212, 2011.

[15] H. K. Nashine and W. Shatanawi, "Coupled common fixed point theorems for a pair of commuting mappings in partially ordered complete metric spaces," Computers \& Mathematics with Applications, vol. 62, no. 4, pp. 1984-1993, 2011.

[16] K. Iseki, "On common fixed points of mappings," Bulletin of the Australian Mathematical Society, vol. 10, pp. 365-370, 1974.

[17] K. Iseki, "Multi-valued contraction mappings in complete metric spaces," Rendiconti del Seminario Matematico della Università di Padova, vol. 53, pp. 15-19, 1975.

[18] A. Rashid Butt, Fixed points of set valued maps [Ph.D. thesis], Department of Mathematics, Lahore University of Management Sciences, Lahore, Pakistan, 2010.

[19] I. Beg and A. R. Butt, "Common fixed point for generalized set valued contractions satisfying an implicit relation in partially ordered metric spaces," Mathematical Communications, vol. 15, no. 1, pp. 65-76, 2010.

[20] S. Karpagam and S. Agrawal, "Best proximity point theorems for P-cyclic Meir-Keeler contractions," Fixed Point Theory and Applications, vol. 2009, Article ID 197308, 9 pages, 2009.

[21] M. De la Sen, "Linking contractive self-mappings and cyclic Meir-Keeler contractions with Kannan self-mappings," Fixed Point Theory and Applications, vol. 2010, Article ID 572057, 23 pages, 2010.

[22] E. Karapinar, "Best proximity points of cyclic mappings," Applied Mathematics Letters, vol. 25, no. 11, pp. 1761-1766, 2012.

[23] M. Jleli, E. Karapinar, and B. Samet, "Best proximity point results for MK- proximal contractions," Abstract and Applied Analysis, vol. 2012, Article ID 193085, 14 pages, 2012.

[24] E. Karapinar, G. Petrusel, and K. Tas, "Best proximity point theorems for KT-types cyclic orbital contraction mappings," Fixed Point Theory, vol. 13, no. 2, pp. 537-545, 2012.

[25] E. Karapinar and I. M. Erhan, "Cyclic contractions and fixed point theorems," Filomat, vol. 26, no. 4, pp. 777-782, 2012.

[26] G. Petrusel, "Cyclic representations and periodic points," Universitatis Babeș-Bolyai. Studia. Mathematica, vol. 50, no. 3, pp. 107-112, 2005.

[27] M. Păcurar and I. A. Rus, "Fixed point theory for cyclic phicontractions," Nonlinear Analysis. Theory, Methods \& Applications A, vol. 72, no. 3-4, pp. 1181-1187, 2010.

[28] V. Pragadeeswarar and M. Marudai, "Best proximity points: approximation and optimization in partially ordered metric spaces," Optimization Letters, 2012.

[29] V. Sankar Raj, "A best proximity point theorem for weakly contractive non-self-mappings," Nonlinear Analysis. Theory, Methods \& Applications A, vol. 74, no. 14, pp. 4804-4808, 2011.

[30] B. Samet, "Some results on best proximity points," Journal of Optimization Theory and Applications, 2013.

[31] M. Kikkawa and T. Suzuki, "Three fixed point theorems for generalized contractions with constants in complete metric spaces," Nonlinear Analysis. Theory, Methods \& Applications A, vol. 69, no. 9, pp. 2942-2949, 2008.

[32] Y. Enjouji, M. Nakanishi, and T. Suzuki, "A generalization of Kannan's fixed point theorem," Fixed Point Theory and Applications, vol. 2010, Article ID 192872, 10 pages, 2009.

[33] A. A. Eldred and P. Veeramani, "Existence and convergence of best proximity points," Journal of Mathematical Analysis and Applications, vol. 323, no. 2, pp. 1001-1006, 2006.
[34] I. A. Pyatyshev, "Operations over approximatively compact sets," Mathematical Notes, vol. 82, no. 5, pp. 729-735, 2007.

[35] B. B. Panda and O. P. Kapoor, "Approximative compactness and continuity of metric projections," Bulletin of the Australian Mathematical Society, vol. 11, pp. 47-55, 1974. 


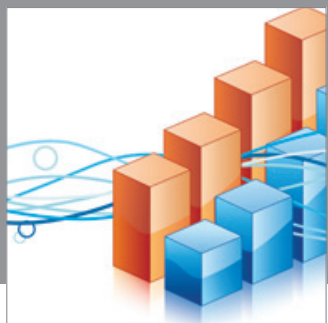

Advances in

Operations Research

mansans

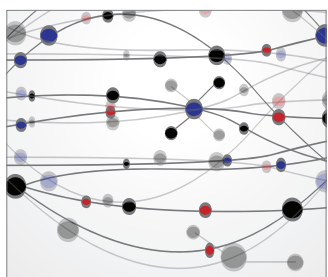

The Scientific World Journal
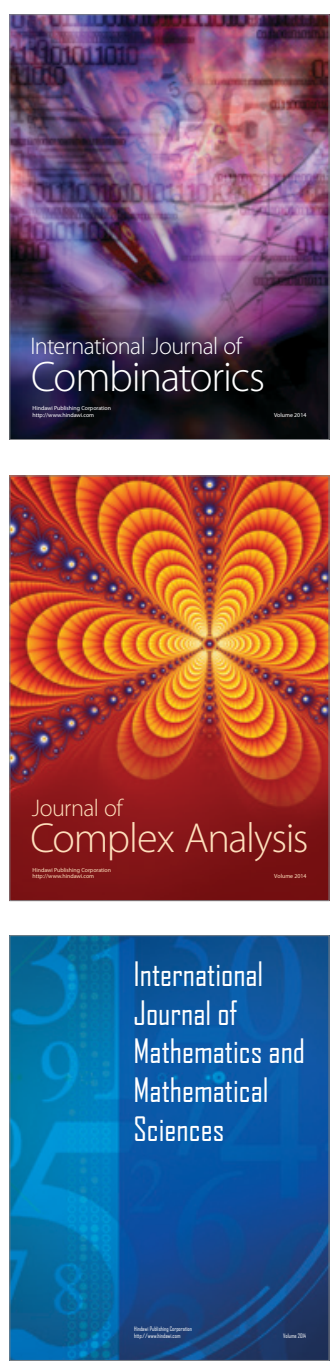
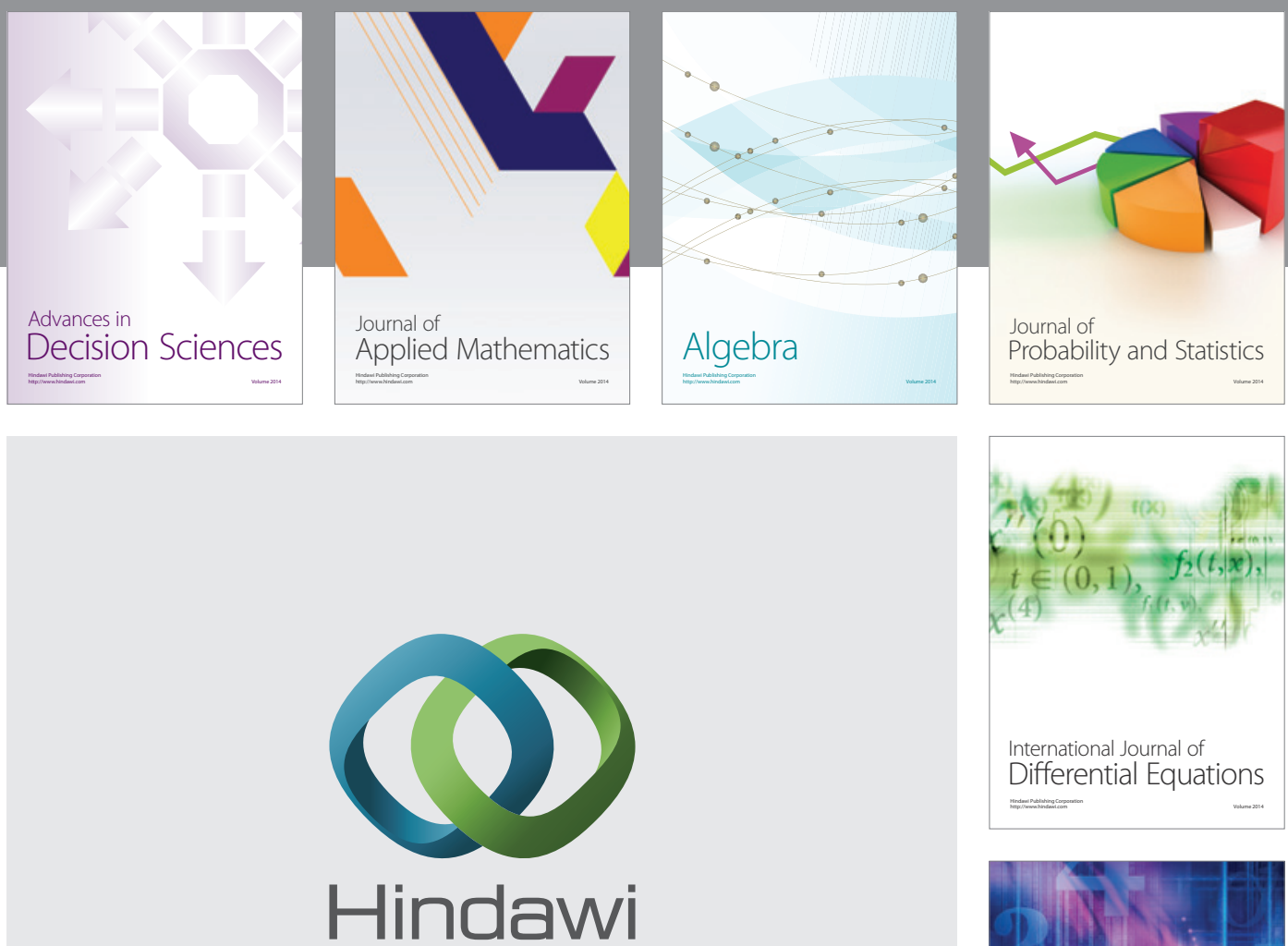

Submit your manuscripts at http://www.hindawi.com
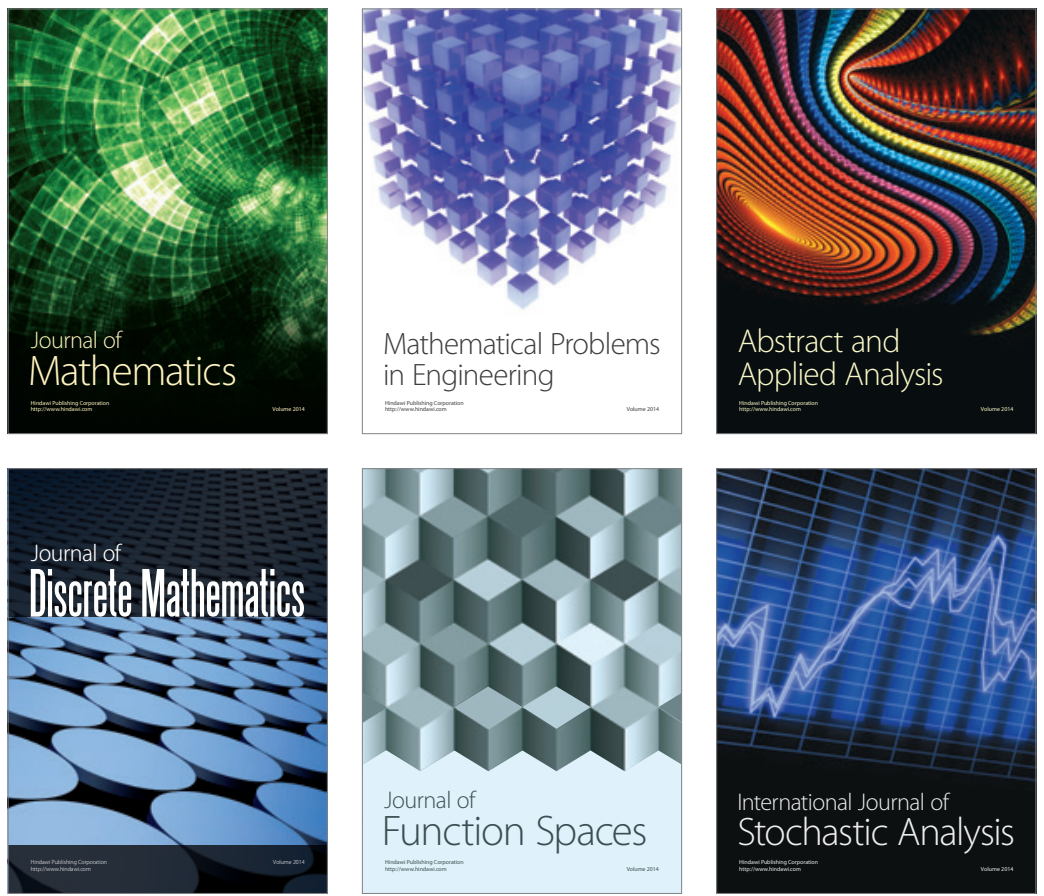

Journal of

Function Spaces

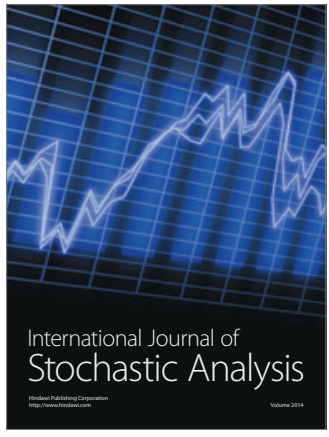

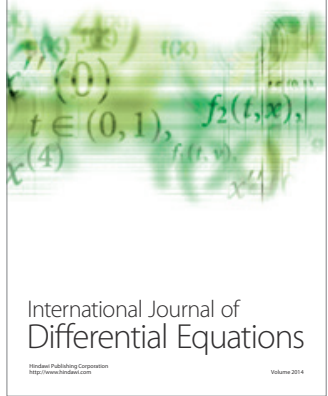
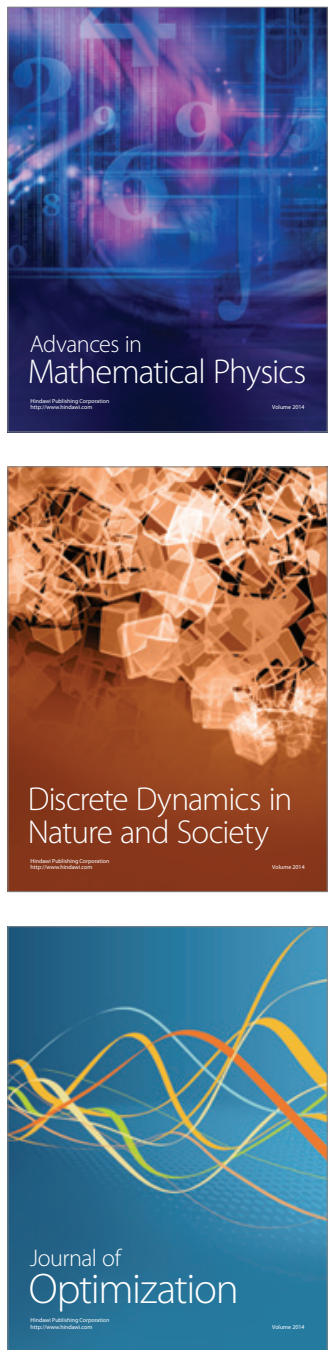